\title{
Hydrogeologie im Tauernfenster - Fallbeispiel Rauristal, Salzburg
}

\author{
Daniel Dirnberger ${ }^{1} \cdot$ Sylke Hilberg ${ }^{2}$
}

Eingegangen: 29. April 2019 / Überarbeitet: 10. Oktober 2019 / Online publiziert: 28. Januar 2020

(c) Der/die Autor(en) 2020

\section{Zusammenfassung}

Die Fallstudie in einem kleinräumigen Einzugsgebiet im Salzburger Rauristal diente dazu, die Hydrogeologie einiger für das Tauernfenster typischer Lithologien und Strukturen beispielhaft zu beleuchten. Das Untersuchungsgebiet ist geprägt von metamorphen geklüfteten Gesteinen des Penninikums und Subpenninikums (Schiefer und Gneise) und quartären Ablagerungen, wobei hier vor allem ein großer Bergsturz als prägendes Element zu nennen ist. Es wurde eine geologisch-hydrogeologische Kartierung des knapp $10 \mathrm{~km}^{2}$ großen Gebietes auf Seehöhen zwischen 1500 und $2500 \mathrm{~m}$ durchgeführt. Im Fokus der Geländearbeiten standen dabei hydrogeologische Aspekte wie Quellen, Vernässungen, Versickerungen oder Oberflächengewässer. Ergänzend wurden an drei Terminen Abflussmessungen am Hauptgerinne und drei seichte Bohrungen in einer Bergsturzmasse durchgeführt, um deren hydraulische Eigenschaften zu erkunden. Es wurden fünf Fließsysteme identifiziert: (1) Schichtquellen sind an den Übergang zwischen Zentralgneis und Schieferhülle gebunden. (2) Stauquellen treten in Verbindung mit Grundmoränen auf. (3) Quellhorizonte innerhalb der Schiefer und Gneise verlaufen entlang regionaler Kluftrichtungen. (4) Diffuse Quellaustritte sind auf oberflächennahe Zirkulationssysteme zurückzuführen. (5) Der Bergsturzkörper stellt einen sehr gut durchlässigen Aquifer dar. Eine Verwitterungsschwarte bildet eine oberflächliche Abdichtung, verbunden mit der Ausbildung zahlreicher kleiner stehender Gewässer.

\section{Hydrogeology of the Tauern window_case study Rauristal, Salzburg}

\begin{abstract}
The aim of this case study in a small-scale catchment at altitudes between 1500 and $2500 \mathrm{~m}$ asl in the Rauristal region in Salzburg was to investigate the hydrogeological character of some typical lithologies and structures along the northern margin of the Tauern Window. The study area is composed of metamorphic schist and gneiss of Penninic and Subpenninic nappes and various Quaternary deposits. Approximately $10 \mathrm{~km}^{2}$ were mapped with regard to geology and hydrogeological aspects such as springs, surface runoff, and sinkholes. Discharge measurements were taken at three dates and three boreholes were drilled to investigate the hydrogeological context of a large landslide deposit. Five different flow systems were identified: (1) Layer-based springs at the boundary between gneiss and schist result from different hydraulic conductivities. While gneiss is a comparably highly permeable fractured aquifer, schists act as aquitards in this setting. (2) Barrier springs are related to moraine deposits. (3) Spring lines within schists follow the main regional fracture direction. (4) Numerous small springs are linked to near surface flow systems within debris layers. (5) The large landslide deposit represents an aquifer with good hydraulic conductivity. Due to weathering layers, this unit is locally impermeable for infiltration which leads to numerous small ponds in the upper part of the landslide deposit.
\end{abstract}

Keywords Fractured rocks · Landslides · Glacial deposits · Tauern Window · Austria

Daniel Dirnberger

daniel.dirnberger@intergeo.com

Sylke Hilberg

sylke.hilberg@sbg.ac.at
1 Intergeo Umwelttechnologie und Abfallwirtschaft $\mathrm{GmbH}$, Robinigstraße 93, 5020 Salzburg, Österreich

2 Fachbereich Geographie und Geologie, Universität Salzburg, Hellbrunner Str. 34, 5020 Salzburg, Österreich 


\section{Einleitung}

Hydrogeologie in alpinen Gebirgsräumen befasst sich vornehmlich mit Festgesteinsaquiferen, die je nach Lithologie entweder verkarstet oder geklüftet auftreten. Grundwasserfluss findet also in Röhrensystemen oder in verzweigten Kluftsystemen unterschiedlichster Größenordnungen oder in einer Kombination aus beidem statt. Welch und Allen (2014) geben einen Überblick über aktuelle Konzepte zum Verständnis von Gebirgsaquiferen und stellen Ansätze zur numerischen Modellierung vor. Viele Konzeptmodelle gehen in nicht verkarstungsfähigen Gebirgsaquiferen von oberflächennahen Fließsystemen aus, die auf die Bodenzone, die Lockergesteinsüberdeckung und die Verwitterungsschwarte des Grundgebirges beschränkt sind (z.B. Fiori et al. 2009; Hilberg und Riepler 2016). Andere Studien (z. B. Banks et al. 2009; Masset und Loew 2010; Hilberg und Kreuzer 2013) zeigen Hinweise darauf, dass auch tiefe Fließsysteme innerhalb des wenig bis nicht verwitterten, dafür aber entsprechend geklüfteten Grundgebirges eine wichtige Rolle für die Hydrodynamik in Gebirgsräumen spielen. Die Lithologie und die tektonische Beanspruchung des Gebirges spielen bei der Ausbildung von tiefgreifenden Aquifersystemen eine bedeutende Rolle. Während kompetente und daher vornehmlich sprödtektonisch deformierte Gesteine wie Granite oder Gneise tiefreichende hydraulisch wirksame Kluftsysteme aufweisen können, sind geschieferte feinkörnige Gesteine, wie Glimmerschiefer als eher gering durchlässig anzusehen.

Störungszonen können sowohl als hydraulische Barriere als auch als bevorzugte Wasserwege fungieren und können zudem erhebliche Anisotropien in ihrer hydraulischen Durchlässigkeit aufweisen (Caine et al. 1996; Winkler et al. 2010; Bense et al. 2013).

Die Lockergesteinsbedeckung der alpinen Festgesteinsaquifere, die auf glaziale, periglaziale, postglaziale und rezente Prozesse zurück geht, bildet häufig lokal gut abgrenzbare Aquifere unterschiedlichster Mächtigkeiten aus. Typisch für Gebirgsräume sind neben rezenten Bildungen wie Hangschuttkörpern, Schwemmfächern, fluviatilen Talfüllungen oder aktiven Massenbewegungen dabei auch aktive, inaktive oder reliktische Blockgletscher, Moränenablagerungen und Ablagerungen von Fels- und Bergsturzereignissen. Hangschuttkörper, Schwemmfächer und fluviatile Talfüllungen können als Porenaquifere beschrieben werden, die aufgrund der Topographie hohe hydraulische Gradienten aufweisen, obwohl häufig eher grobe Korngrößen und damit hohe Durchlässigkeiten vorliegen. Tiefgreifend aufgelockerte Massenbewegungen an übersteilten Hängen bieten gute Durchlässigkeiten entlang der Bewegungsbahnen oder komplexe Fließsysteme durch die interne Auflockerung der Rutsch- oder Kriechmassen (Ronchetti et al. 2009; Strauhal et al. 2015; Vallet et al. 2015). Blockglet- scher zeigen häufig eine interne Struktur, in der sich blockige grobe Komponenten mit feinkörnigeren Schichten abwechseln, was zu einem komplexen Fließgefüge mit kurzen bis mittleren Verweilzeiten und damit schnellen und langsamen Fließkomponenten führt (Winkler et al. 2016; Pauritsch et al. 2017; Jones et al. 2019). Moränenablagerungen sind aufgrund ihrer schlechten Sortierung und ihres hohen Feinkornanteils, Grundmoränen zudem aufgrund der hohen Lagerungsdichte gering durchlässig und bilden damit lokale Stauhorizonte aus. Ablagerungen, die aus Fels- oder Bergstürzen resultieren, bilden im alpinen Raum lokal begrenzte, oft mächtige und vorwiegend grobblockige Lockergesteinskörper, für die generell sehr gute Durchlässigkeiten erwartet werden. Obwohl derartige Bergsturzmassen in Folge spätglazialer Druckentlastungen (z. B. Reitner et al. 1993) im Alpenraum häufig auftreten, wurde ihre hydrogeologische Charakteristik bisher nur wenig untersucht. Lauber et al. (2014) beschreiben beispielsweise die gute Durchlässigkeit und hohe Fließgeschwindigkeiten innerhalb einer Bergsturzmasse im bayerischen Wettersteingebirge.

Die hier präsentierte Arbeit im Salzburger Rauristal stellt eine Fallstudie im Projektraum dar. Aufgrund der geologischen Situation im Untersuchungsgebiet, die auf kleinem Raum viele der typischen geologischen Elemente der zentralen Ostalpen vereint, lassen sich die Ergebnisse und hydrogeologischen Interpretationen aber auf andere Bereiche des Tauernfensters übertragen. In dem kleinräumigen Untersuchungsgebiet soll beispielhaft gezeigt werden, welches Spektrum an Fließsystemen auftreten kann und ob und wie sich Quellen aus oberflächennahen Porenaquiferen von tiefreichenden Festgesteinsaquiferen unterscheiden lassen. Ergänzend soll beispielhaft die hydrogeologische Charakteristik einer Bergsturzmasse beleuchtet werden.

\section{Untersuchungsraum}

Das Untersuchungsgebiet liegt im Talschluss des Rauristals (Kolm-Saigurn) im Bundesland Salzburg in Österreich. Es ist Teil des Nationalparks Hohe Tauern und liegt innerhalb der Goldberg-Gebirgsgruppe. Das etwa $60 \mathrm{~km}$ südlich der Stadt Salzburg gelegene Rauristal gehört mit einer Länge von $30 \mathrm{~km}$ zu den längsten glazial ausgeformten Trogtälern der Tauern. Der Untersuchungsraum erstreckt sich auf Höhenlagen zwischen 1500 und $2500 \mathrm{~m}$ ü. A. (Abb. 1). Es wurde eine Fläche von knapp $10 \mathrm{~km}^{2}$ detailliert geologisch und hydrogeologisch kartiert.

Das Gebiet ist vor allem für seine jahrhundertealte Bergbaugeschichte bekannt. Älteste urkundliche Erwähnungen des Bergbaus finden sich ab dem späten 13. Jh. (Günther und Paar 2000). Die Blüte des vornehmlich auf Gold ausgerichteten Bergbaus begann aber im 14. Jh. und endete in den 1930er Jahren. Bei den Goldlagerstätten handelt es sich 


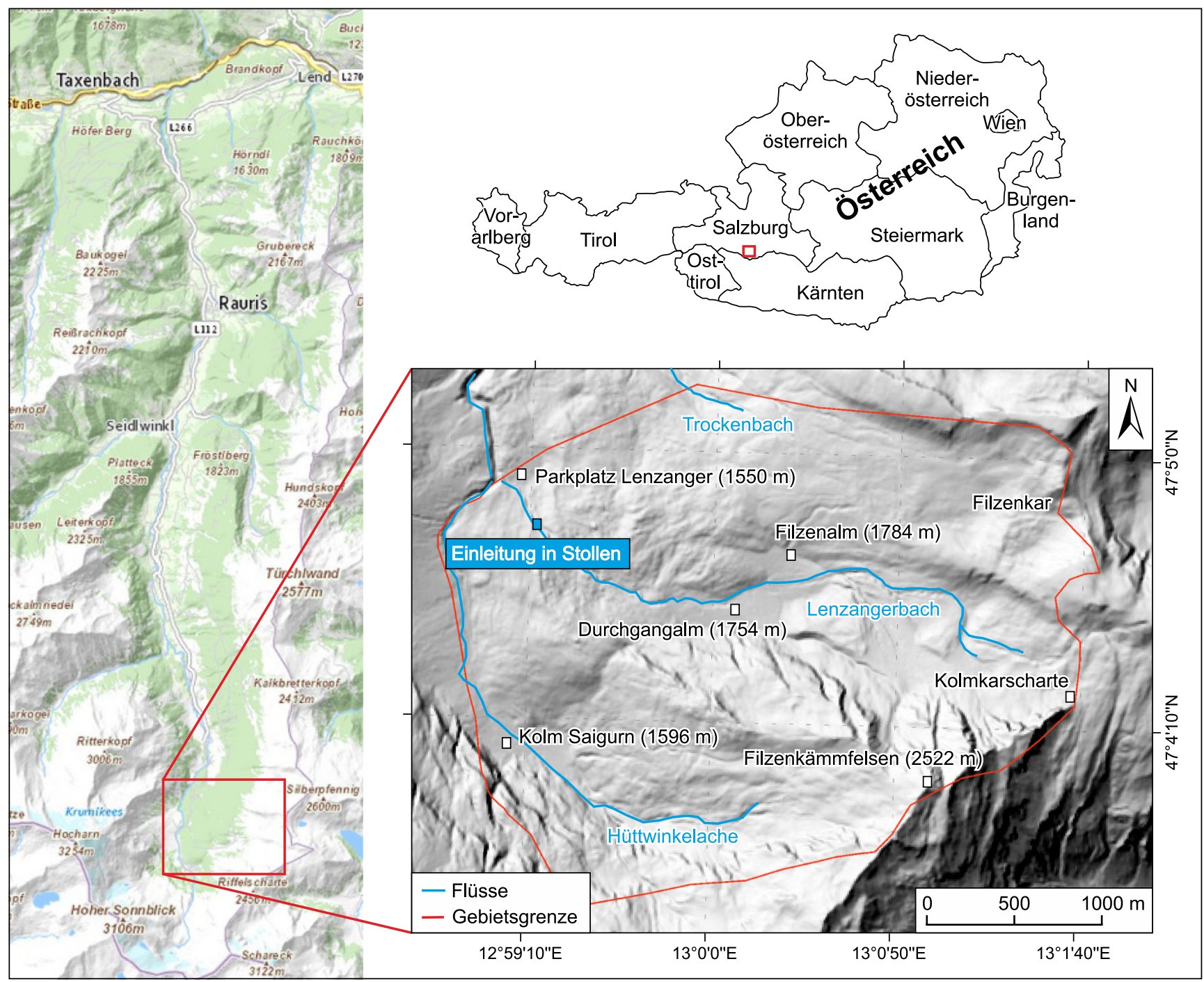

Abb. 1 Lage und Übersicht über das Untersuchungsgebiet (Kartengrundlagen Land Salzburg 2014)

Fig. 1 Location and overview of the study area

um hydrothermale Gänge, die sogenannten Tauergoldgänge (TGG), die an NNE-streichende Kluftsysteme gebunden sind (Paar et al. 2006). Aus der Zeit des Bergbaus existieren noch zahlreiche kleinere Stollensysteme und Halden im hinteren Rauristal. Direkt im Untersuchungsgebiet verläuft der Imhofstollen. Dieser wurde als Aufschluss- und Förderstollen am Beginn des 20. Jahrhunderts errichtet und unterfährt das Untersuchungsgebiet zwischen Hüttwinkeltal und Nassfeld.

Ebenfalls in diesem Bereich unterfährt ein Überleitungsstollen das Untersuchungsgebiet. Hier wird Wasser der Hüttwinklache sowie des Lenzangerbaches für die Stromerzeugung zum Speicher Bockhartsee abgeleitet (Salzburg AG 2018).

Das Gebiet befindet sich am Nordrand des Tauernfensters, dem größten tektonischen Fenster innerhalb der Ostalpen, in dem die subpenninischen und penninischen Ein- heiten, die ansonsten unter den ostalpinen Deckenstapeln lagern, aufgeschlossen sind (Pestal et al. 2009; Schmid et al. 2013). Seit der ersten Hälfte des 20. Jahrhunderts wurden hier geologische Untersuchungen durchgeführt, die sich einerseits mit dem geologisch-tektonischen Aufbau des Gebirges (Hottinger 1935; Exner 1957; Matura 1966; Alber 1998), andererseits auch mit den hier anzutreffenden Massenbewegungen und quartären Ablagerungen (von Poschinger 1986; Fellner 1993; Bichler et al. 2016) befasst haben.

In den oberen, zum Teil sehr steilen Hanglagen des hinteren Rauristales (Hüttwinkltal) sind Festgesteine des Penninikums (Bündnerschiefergruppe) und des Subpenninikums (helle und dunkle Glimmerschiefer, Granatglimmerschiefer) sowie im nordöstlichen Abschnitt Gneis aufgeschlossen. 
Die hydrogeologisch relevante Störungszone (Hobiger und Kollmann 2006; Hilberg et al. 2013) zwischen den Schiefern und dem Zentralgneis ist hauptsächlich von mächtigen Blockschuttmassen überdeckt und nur im nördlichen Bereich des Untersuchungsraumes kleinräumig direkt aufgeschlossen. Große Bereiche des Untersuchungsraumes sind mit quartären Sedimenten bedeckt, die im Detail von Bichler und Reindl (2013) quartärgeologisch untersucht worden sind.

Ca. $3 \mathrm{~km}^{2}$ der Fläche bildet der sogenannte Durchgangwald, der aufgrund seiner dichten Bewaldung sowie einer Vielzahl großer moosbewachsener Sturzblöcke auch als „Rauriser Urwald“ bezeichnet wird. Hottinger (1935) und später dann von Poschinger (1986) deuteten die Masse als Großbergsturz, dessen Liefergebiet an der westlichen Talflanke in einer deutlichen Hohlform am GrieswiesSchwarzkogel zu finden ist. Bichler et al. (2016) fanden innerhalb der Bergsturzablagerung eine interne Schichtung, die der inversen Schichtabfolge des gegenüberliegenden Hanges des Grieswies-Schwarzkogel entspricht und von matrixdominierten feinkörnigen glazialen Sedimenten überlagert wird. Sie führten Datierungen an Sturzblöcken durch, die zeigen, dass dem Bergsturz vor $13 \pm 1,1 \mathrm{ka}$ ein rascher Gletschervorstoß folgte. Die glazialen Sedimente wurden bereichsweise anschließend nochmals von einem kleineren Bergsturz (10,8 $\pm 1,1 \mathrm{ka})$ überdeckt.

Der Lenzangerbach, der Trockenbach und die als Hauptvorflut des hinteren Rauristales wirkende Hüttwinklache entwässern das Gebiet.

\section{Methodik}

Im Zeitraum Juni bis September 2014 wurde eine geologisch-hydrogeologische Kartierung im Maßstab 1:10.000 durchgeführt. Dabei wurden die Verbreitung der Gesteinstypen, Gefügedaten sowie hydrogeologisch relevante Aufschlusspunkte, wie Quellen, Vernässungen und Oberflächengewässer detailliert erfasst, digital verortet und in ein Kartenblatt überführt. Die Gefügedaten wurden mithilfe des Programms Open Stereonet verarbeitet und ausgewertet. An Quellen und Oberflächengewässern wurden die Feldparameter Temperatur und elektrische Leitfähigkeit mithilfe eines TechProII TPh der Firma MyronL erfasst. Schüttungsmengen von Quellen wurden mittels Gefäßmessung ermittelt. An den Oberflächengewässern wurden Abflussmessungen nach der Salzverdünnungsmethode unter Einsatz des Messsystems TQ-Trace der Firma Sommer Messtechnik durchgeführt. Der Abfluss des Lenzangerbaches, als zentraler Hauptabfluss des Untersuchungsgebietes, wurde an mehreren Stellen im Fließverlauf und bei augenscheinlich unterschiedlichen Fließmengen gemessen, um die $\mathrm{Zu}$ - und Abflussbedingungen innerhalb der
Bergsturzmasse zu erfassen. Im Bereich Durchgangwald wurde der oberflächennahe geologische Aufbau mithilfe von Handkernbohrungen bis in eine Tiefe von maximal $60 \mathrm{~cm}$ erkundet, um die zahlreich vorhandenen Stillgewässer sowie das Schüttungsverhalten des Lenzangerbaches mit den von Bichler et al. (2016) postulierten spätglazialen Ablagerungen in Beziehung zu setzen.

\section{Ergebnisse}

\section{Geologie/Strukturgeologie}

Die Geologie im Untersuchungsgebiet (Abb. 3) wird geprägt von Gesteinen des Penninikums und des Subpenninikums des Tauernfensters. Der Zentralgneis wird hier von einem Granitgneis repräsentiert, dessen charakteristisches Merkmal einige Zentimeter große Kalifeldspäte darstellen und der daher als Augengneis bezeichnet wird. Er tritt in wenigen Aufschlüssen im nordöstlichen Bereich des Kartiergebietes zwischen Bockhartscharte, Filzenkar und Filzenalm auf, ist aber meist von Hangschuttmassen überdeckt.

Die auf dem Zentralgneis tektonisch auflagernde Venedigerdecke ist durch Glimmerschiefer der Habachgruppe vertreten. Die Variationen reichen von grünlichen ChloritSerizitschiefern und Schwarzschiefern über silbrige Phyllite bis hin zu stark granatführenden Glimmerschiefern, die im Foto in Abb. 2 dargestellt sind. Immer wieder sind auch geringmächtige Karbonatlagen parallel zur Schieferung eingeschaltet. Anstehende Kalkglimmerschiefer wurden lediglich in den topographisch höchsten Bereichen um den Filzenkämmfelsen angetroffen.

Im Kartenmaßstab lassen sich hellere und dunklere Glimmerschiefer unterscheiden, wobei die dunklen Glimmerschiefer eher in den höheren Lagen im Osten aufgeschlossen sind und die hellen Glimmerschiefer die topographisch tieferen Bereiche aufbauen.

Das Einfallen der Schieferungsflächen ist im gesamten Bereich mit etwa 20 bis $40^{\circ}$ flach bis mittelsteil nach SW bis WSW gerichtet. Eine Hauptkluftrichtung bilden NNW- bis NNE-streichende Klüfte, die mit $70^{\circ}$ bis $90^{\circ}$ einfallen. Diese verläuft damit parallel zu den z.B. von Paar et al. (2006) beschriebenen Tauerngoldgängen. Eine zweite Kluftschar streicht ENE und zeigt ähnlich steil stehende Klüfte.

Der Untersuchungsraum weist für das alpine Umfeld typische glaziale, spät- und postglazialen Ablagerungen auf. Grundmoränen überdecken den größten Bereich in den mittleren Höhenlagen des Untersuchungsgebietes. Sie zeichnen sich durch ein weites Korngrößenspektrum, polymikte Zusammensetzung (lt. Bichler und Reindl (2013) vor allem Biotitschiefer und Gneiskomponenten), kantige Komponenten und hohe Lagerungsdichte aus. Markante 


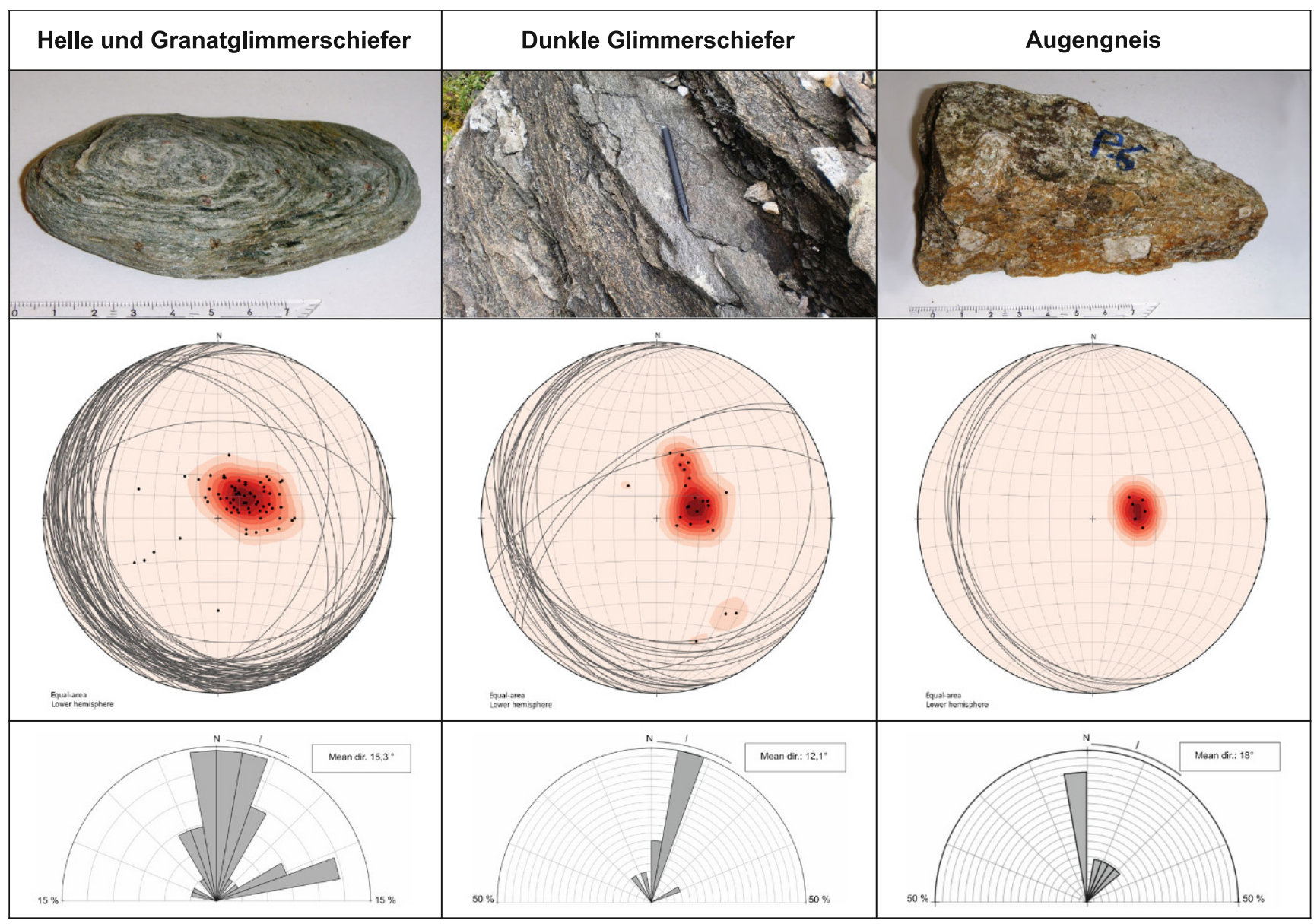

Abb. 2 Fotos der auskartierten Lithologien, Projektion der Schieferungsflächen mit entsprechenden Polpunkten im Schmidtschen Netz und Darstellung der Hauptkluftrichtungen in den drei Lithologien

Fig. 2 Images of the mapped lithologies, layer and joint orientation

Seitenmoränenwälle finden sich vor allem oberhalb der Filzenalm. Das Filzenkar ist von grobblockigem Material eines fossilen Blockgletschers mit wulstförmiger Front bedeckt. Blockiger Hangschutt bedeckt vor allem die mittelsteilen oberen Hangabschnitte im Osten und Süden des Untersuchungsraumes. Die Verebnungsflächen der Filzenalm werden von fluviatilen Ablagerungen des Lenzangerbaches gebildet. Hier finden sich mindestens zwei Terrassenstufen früherer Talniveaus.

Das markanteste quartäre Element im Projektgebiet ist der Großbergsturz, der im NW von der Filzenalm bis nahezu zum Boden des Hüttwinkltales reicht. Er hat eine flächige Ausdehnung von ca. $3 \mathrm{~km}^{2}$ und wird auf ein Volumen von ca. $0,4 \mathrm{~km}^{3}$ geschätzt (Bichler et al. 2016), erreicht damit also im Schnitt mehr als $100 \mathrm{~m}$ Mächtigkeit. Die bereits von von Poschinger (1986) und Bichler et al. (2016) festgestellte interne Schichtung entsprechend der inversen Schichtlagerung im gegenüberliegenden Liefergebiet konnte auch im Rahmen dieser Kartierung nachvollzogen werden. Auf Basis der oberflächlich angetroffenen Abfolge lässt sich schließen, dass Bergsturzmaterial aus vorwiegend hellen
Glimmerschiefern die dunklen Glimmerschiefer überlagert, die wiederum Kalkglimmerschiefern auflagern.

\section{Hydrogeologie}

Im Untersuchungsgebiet wurden insgesamt 211 Quellaustritte (Abb. 3) sowie 50 kleine teilweise verlandete Stillgewässer und Vernässungszonen erfasst. Die drei Hauptabflüsse Hüttwinklache im Süden, Lenzangerbach im zentralen Bereich des Untersuchungsraumes und Trockenbach im Norden verlaufen nach NW und werden von dendritisch ausgeprägten oberflächlichen Entwässerungsstrukturen gespeist. Die Schüttungsmengen der meisten Quellen sind mit Stichtagswerten zwischen 0,01-0,61/s gering. Nach längeren Trockenphasen fallen viele dieser kleinen Quellen trocken.

Hinsichtlich der räumlichen Verteilung der Quellen fallen Quellhorizonte auf, die nach NNE ausgerichtet sind und damit einer der Hauptkluftrichtungen im Gebiet folgen.

Die für nicht verkarstete Kluftaquifere sehr großen Schüttungsmengen einiger Quellen am Westrand der 


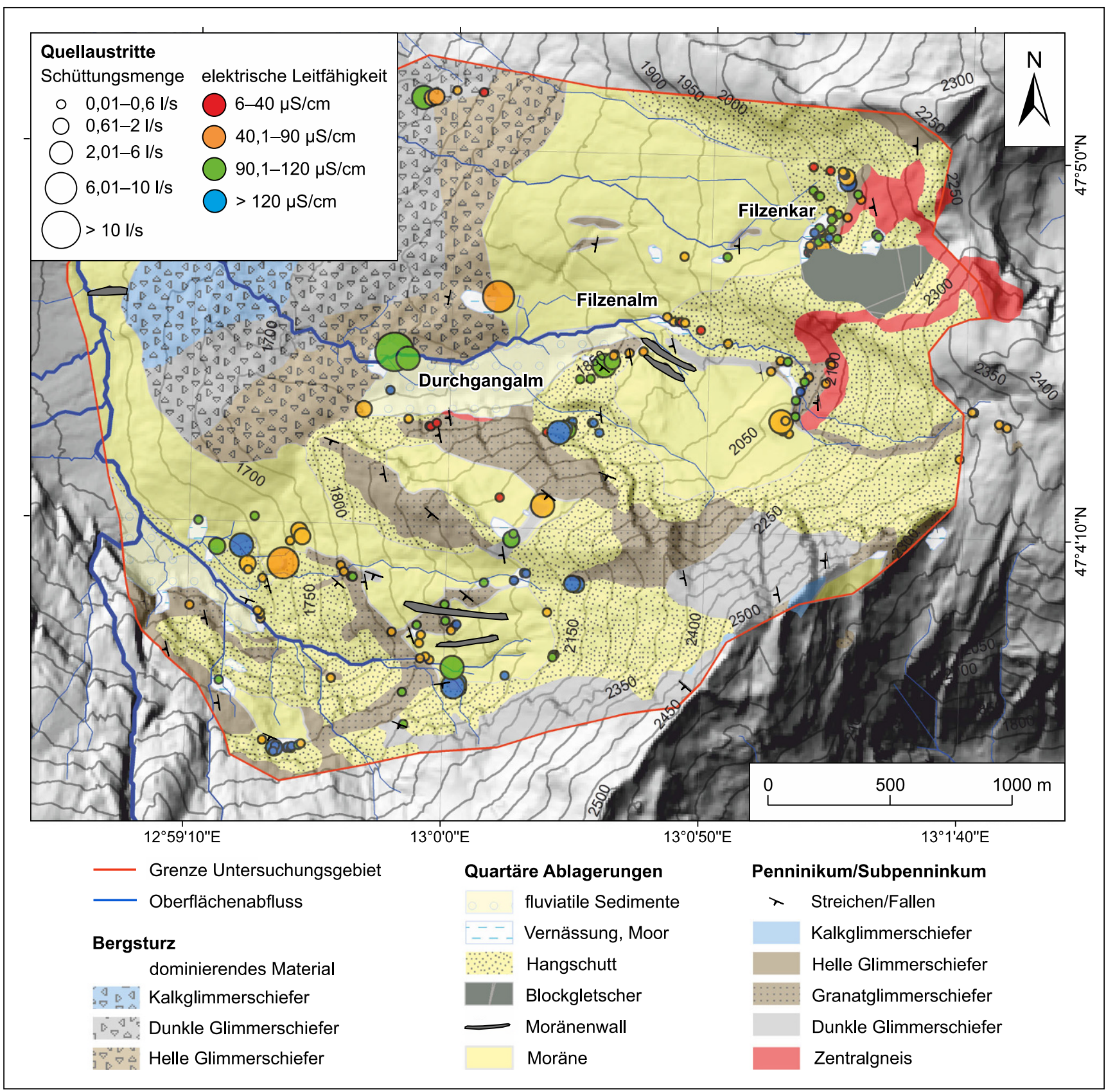

Abb. 3 Hydrogeologische Karte, Quellaustritte, klassifiziert nach Schüttungsmenge und elektrischer Leitfähigkeit

Fig. 3 Hydrogeological map, springs classified according to discharge and electrical conductivity

Durchgangalm von bis zu 251/s, sind auf Umläufigkeiten des Lenzangerbaches zurückzuführen, der im Bereich Filzenalm innerhalb gut durchlässiger fluviatiler Sedimente fließt, hier auf ca. $200 \mathrm{~m}$ Fließstrecke in den Porenaquifer infiltriert und beim Eintritt des Bachlaufs in den Bergsturzbereich als Quellgruppe wieder austritt. Dies ließ sich augenscheinlich aus den während der Geländeaufnahmen in der betroffenen Fließstrecke deutlich geringeren Schüttungsmengen im Bachbett ableiten.
Es lassen sich auf Basis des Geländebefundes sowie der Lage der Quellaustritte in Bezug zur geologischen Situation im Gebiet vier Typen von Quellaustritten unterscheiden (Abb. 4):

1. Stauquellen treten im Übergangsbereich von gut durchlässigem Hangschutt zur Grundmoräne auf, die als Geringleiter einzustufen ist. Der bedeutendste zusammenhängende Quellhorizont dieser Art befindet sich im Filzenkar am Fuß des hier ausgeprägten fossilen Blockglet- 


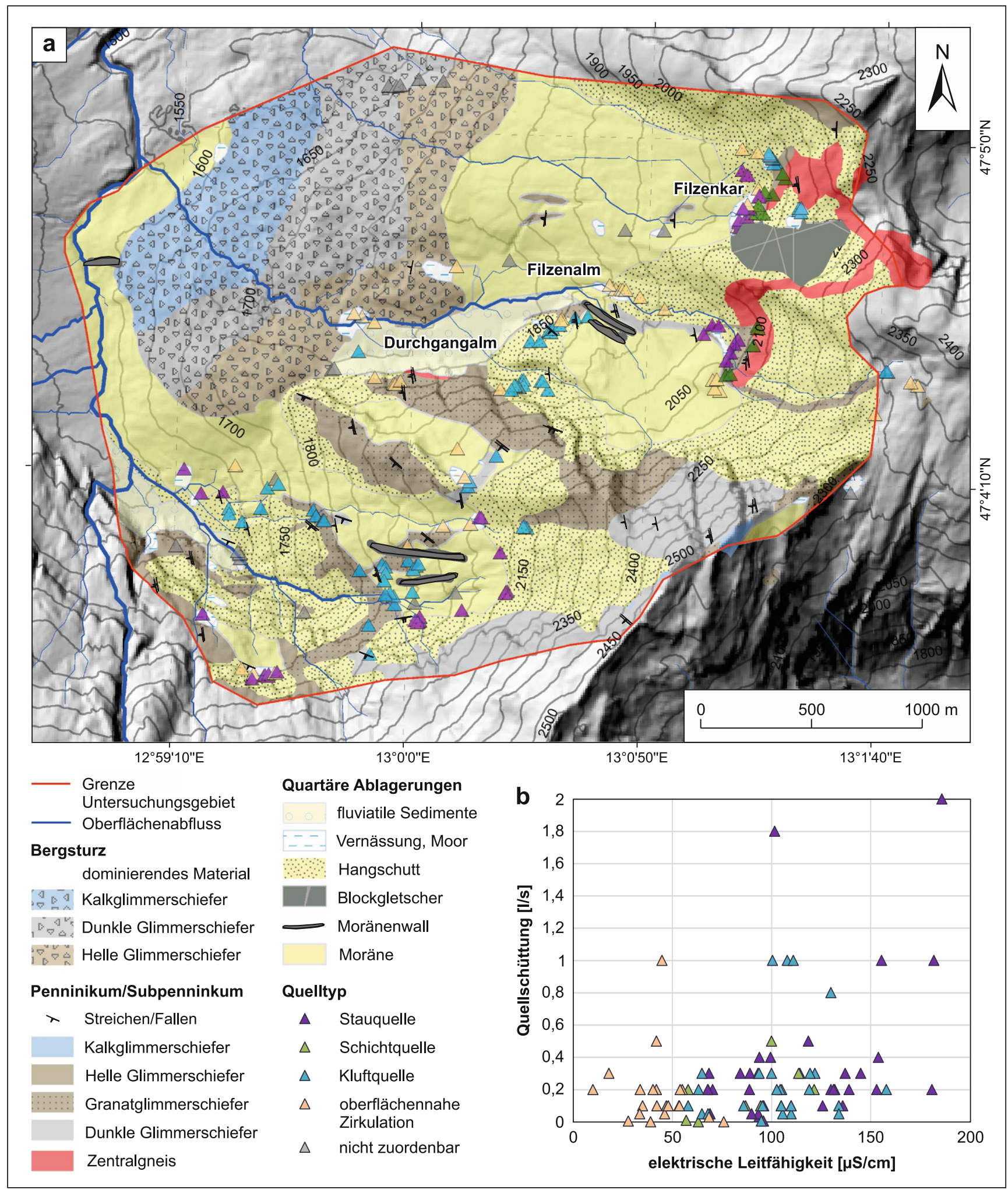

Abb. 4 Quelltypen im Untersuchungsgebiet; a hydrogeologische Karte mit Zuordnung der Quelltypen auf Basis des Geländebefundes; neben der geologischen Situation am Quellaustritt wurden zur Klassifizierung Feldparameter herangezogen; b Schüttungsmengen und elektrische Leitfähigkeiten der Quellen, für die eine eindeutige Zuordnung zu einem Quelltyp möglich war

Fig. 4 Spring classification in the study area; a hydrogeological map with spring classification based on the geological conditions at the spring outflow and further field data; $\mathbf{b}$ discharge and electrical conductivity of springs 


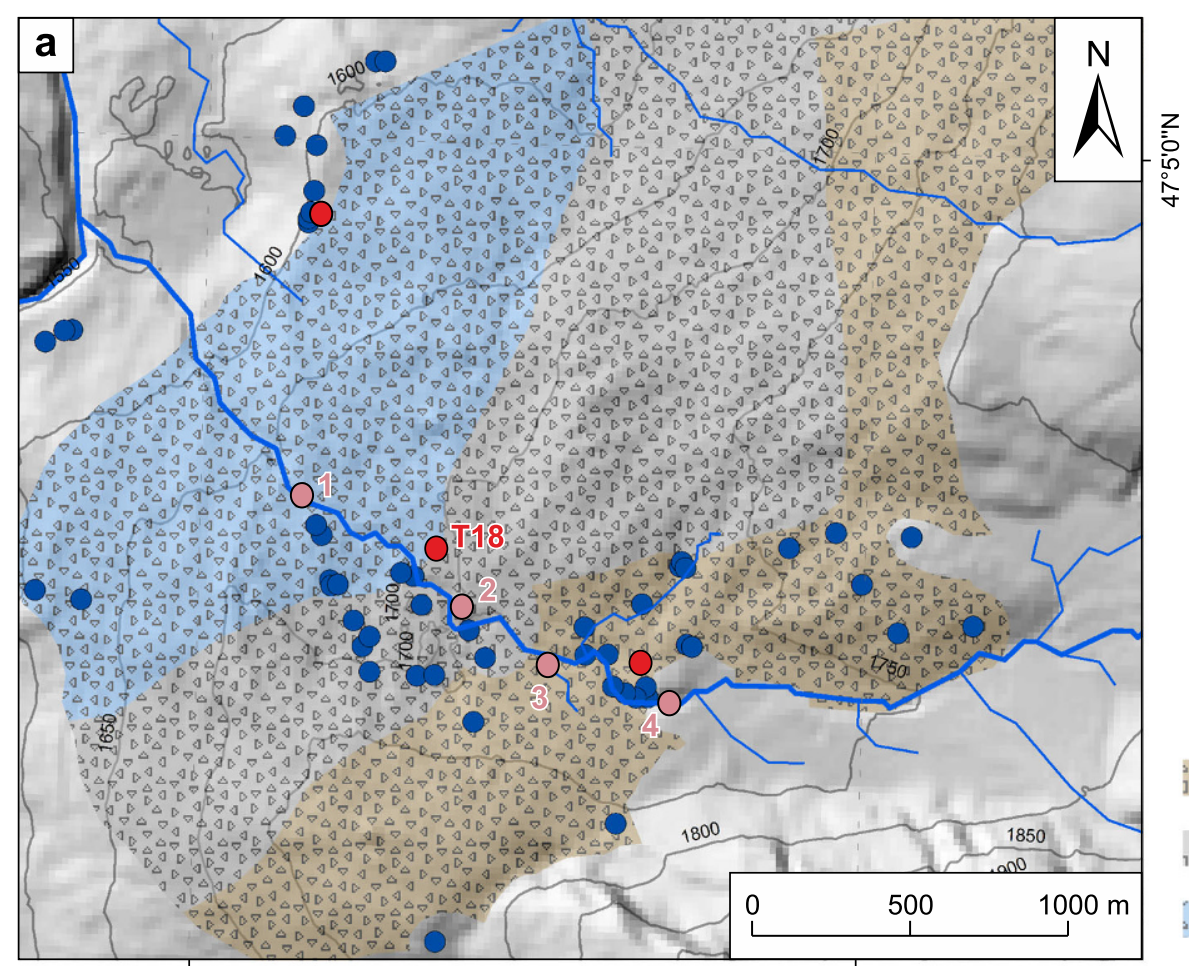

$12^{\circ} 59^{\prime} 10^{\prime \prime} \mathrm{E}$

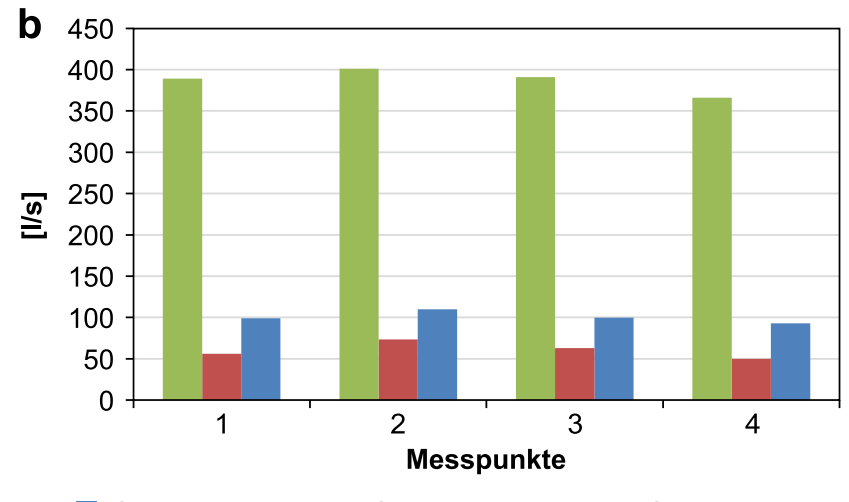

Q-Mittelwasser Q-Niederwasser Q-Hochwasser

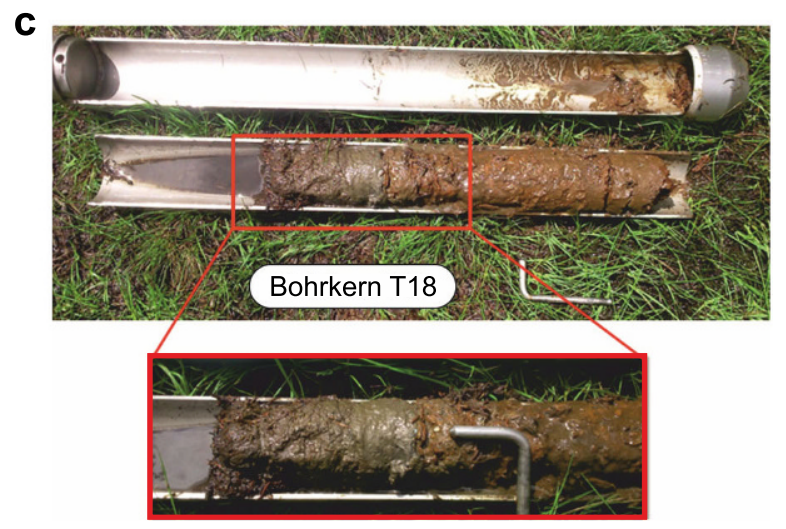

d Messpunkt 4

SE

Stillgewässer

Bohrpunkt

Messpunkt Abflussmessung

$\nabla \nabla * \quad$ Bergsturzmaterial helle Glimmerschiefer

D. Bergsturzmaterial dunkle Glimmerschiefer

(1) 4 Bergsturzmaterial Kalkglimmerschiefer

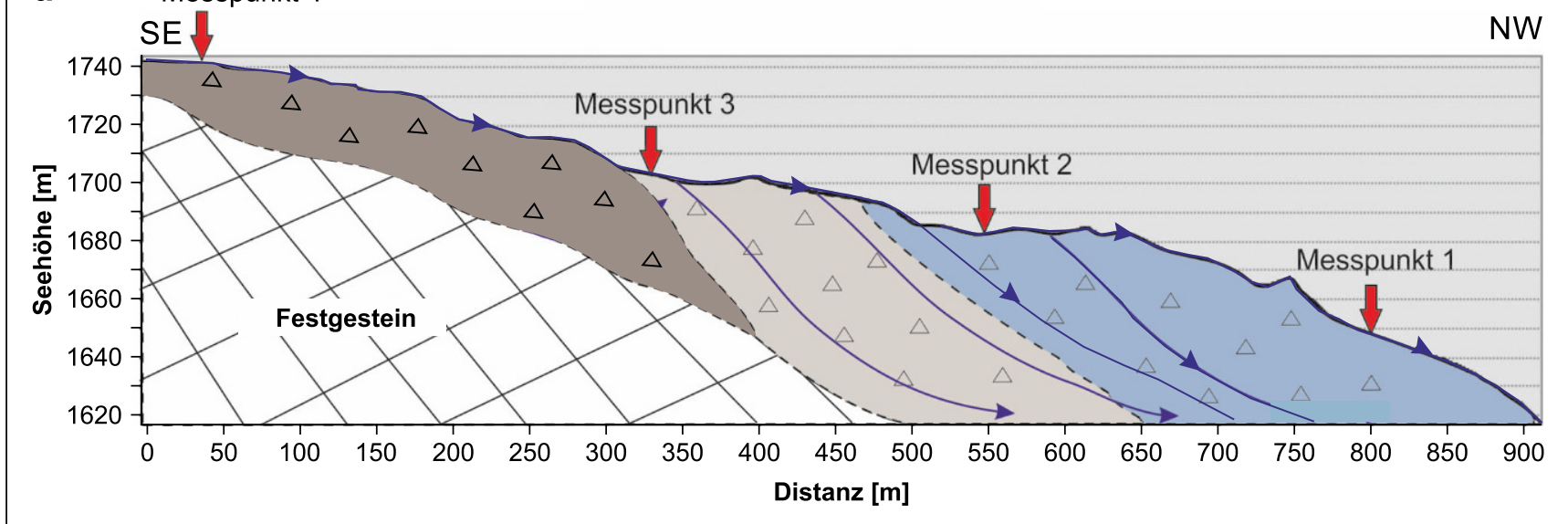


Abb. 5 a Geologische Karte der Bergsturzablagerung mit Stillgewässern, Bohrpunkten und Messpunkten der Abflussmessung; b Schüttungsmengen des Lenzangerbaches, ermittelt zu drei verschiedenen Abflusssituationen; c Verwitterungsschwarte in Bergsturzmaterial; d Bachprofil mit Messpunkten und hydrogeologisches Konzeptmodell des Bergsturzes

Fig. 5 a Geological map of the landslide deposit with ponds, drilling locations and discharge measurement points; $\mathbf{b}$ discharge of Lenzangerbach at three different runoff conditions; $\mathbf{c}$ weathering layer within the landslide deposit; $\mathbf{d}$ cross-section along the brook with measuring points and conceptual hydrogeological model of the landslide

schers. Quellen, die diesem Typus zuzuordnen sind, treten entlang von Horizonten jeweils am Fuß der Schuttkörper aus. Sie weisen meist geringe Schüttungsmengen von unter 0,5 1/s. Einzelne Quellaustritte zeigen aber auch Schüttungsmengen bis $21 / \mathrm{s}$. Es zeigen sich hier mittlere bis hohe elektrische Leitfähigkeiten zwischen $100 \mu \mathrm{S} / \mathrm{cm}$ und $190 \mu \mathrm{S} / \mathrm{cm}$. Die Temperaturen variieren in Abhängigkeit von der Höhenlage des Austritts zwischen 4 und $10^{\circ} \mathrm{C}$. Die geringsten Temperaturen zeigen dabei erwartungsgemäß jene Quellen, die in den höchsten Bereichen, z. B. am Fuß des Blockgletschers austreten.

2. Schichtquellen treten entlang der Grenzschicht zwischen zwei unterschiedlich durchlässigen Festgesteinen auf. Dieser Quelltyp ist im Untersuchungsgebiet deutlich entlang der Grenze zwischen Schiefer und Zentralgneis ausgeprägt. Vergleichsweise hoher Kluftporosität des Zentralgneises steht eine deutlich verringerte Durchlässigkeit der Glimmerschiefer gegenüber. Diese Tatsache konnte lediglich im Bereich des Filzenkars, nördlich des Blockgletschers direkt verifiziert werden. Die Störungszone zwischen diesen beiden Schichten ist im Großteil des Untersuchungsgebietes von quartären Ablagerungen überdeckt. Daher lassen sich deren hydraulische Eigenschaften aus der Kartierung nur bedingt direkt ableiten. Quellhorizonte innerhalb der Hangschuttbedeckung lassen jedoch zusätzlich darauf schließen, dass diese mit der tektonischen und lithologischen Grenze in Zusammenhang stehen. Die Quellen zeigen geringe Schüttungsmengen unter $0,5 \mathrm{l} / \mathrm{s}$ und mittlere elektrische Leitfähigkeiten zwischen $40 \mu \mathrm{S} / \mathrm{cm}$ und $120 \mu \mathrm{S} / \mathrm{cm}$ und Temperaturen zwischen 5 und $8^{\circ} \mathrm{C}$.

3. Kluftquellen sind an den Ausbiss von Klüften an der Oberfläche gebunden und treten im gesamten Untersuchungsraum in Abhängigkeit von Kluftstrukturen auf. In weiten Bereichen sind diese Kluftstrukturen zwar von Hangschutt überdeckt, die Ausrichtung der Quellhorizonte entsprechend der Hauptkluftrichtung kombiniert mit mittleren bis hohen elektrischen Leitfähigkeiten von $40 \mu \mathrm{S} / \mathrm{cm}$ bis $160 \mu \mathrm{S} / \mathrm{cm}$, mit 4 bis $6^{\circ} \mathrm{C}$ vergleichsweise niedrigen Temperaturen sowie Schüttungsmengen bis 11/s lassen aber darauf schließen, dass die Wässer auch im unterlagernden Glimmerschiefer zirkuliert sind und dabei hydrochemisch beeinflusst wurden.

4. Eine große Anzahl von Quellen lässt sich als Schuttquellen charakterisieren, deren Wässer ausschließlich kurzfristig innerhalb der Hangschuttmassen zirkulieren. Sie treten diffus im gesamten Untersuchungsraum auf, weisen geringe elektrische Leitfähigkeiten von meist unter $40 \mu \mathrm{S} / \mathrm{cm}$ auf. Die Temperaturen variieren hier stark mit der Höhenlage des Austritts und lagen während der Feldarbeiten zwischen 4 und $12{ }^{\circ} \mathrm{C}$. Es handelt sich um Quellaustritte mit meist geringer Schüttung $(<0,11 / \mathrm{s})$, die nach längeren Trockenperioden trockenfallen. Einzelne Quellen, die sehr große Schüttungsmengen aufweisen, sind auf Umläufigkeiten der Fließgewässer zurückzuführen, wie das Beispiel der Quellgruppe am Westrand der Durchgangalm zeigt.

Der Bergsturz im Durchgangwald ist charakterisiert durch eine Vielzahl an kleinen stehenden Gewässern und Vernässungen (Abb. 5a). Die Wässer in den Stillgewässern weisen extrem geringe elektrische Leitfähigkeiten von weniger als $15 \mu \mathrm{S} / \mathrm{cm}$, während der sommerlichen Stichtagsmessung Temperaturen von über $10^{\circ} \mathrm{C}$ und leicht saure pH-Werte zwischen 5 und 6 auf. Alle Felddaten zeigen damit, dass es sich um Niederschlagwässer handelt, die hier gesammelt werden und nur sehr langsam in die grobblockige Bergsturzablagerung infiltrieren. In einigen Bereichen kommt es auch zur Entstehung kleiner Hochmoore. Handbohrungen im Randbereich dreier Stillgewässer zeigen eine mindestens $60 \mathrm{~cm}$ mächtige Ton- bis Schluffschicht (Abb. 5c), die ein Versickern der Regenwässer in diesen Bereichen deutlich retardiert. Es muss jedoch ein unterirdischer Abfluss stattfinden, da keine Aufkonzentration der Wässer durch Verdunstung zu beobachten ist.

Abflussmessungen entlang des Lenzangerbaches, der den einzigen Oberflächenabfluss innerhalb des Bergsturzes darstellt, zeigen deutliche Variationen der Durchlässigkeit zwischen Durchgangalm und Talboden. Während die Schüttung im oberen Gewässerabschnitt, in dem sich die meisten Stillgewässer befinden, bei Nieder-, Mittel- und Hochwasser zunächst zunimmt, verliert der Bach in den tieferen Abschnitten jeweils deutlich, dotiert also den Grundwasserkörper innerhalb der Bergsturzmasse. Abb. 5b zeigt die Ergebnisse der Schüttungsmessungen. Das daraus abgeleitete hydrogeologische Konzeptmodell für die Bergsturzmasse ist Abb. 5d zu entnehmen.

\section{Zusammenfassung und Interpretation}

Das Gebiet zeigt mit 211 aufgefundenen Quellaustritten auf einer Fläche von knapp $10 \mathrm{~km}^{2}$ eine hohe Quelldichte. Anhand der Feldparameter und hier vor allem der elek- 
trischen Leitfähigkeiten in Kombination mit der geologischen Situation lassen sich die Quellen klassifizieren. Das Spektrum an Quelltypen umfasst oberflächennahe Schuttquellen inklusive der Umläufigkeiten von Fließgewässern und Stauquellen am Übergang zwischen gut und weniger gut durchlässigen Lockergesteinen. Daneben wurden aber auch Schicht- und Kluftquellen identifiziert, also Wässer, die nicht nur in der quartären Überdeckung sondern auch im Festgestein zirkulieren. Die physiko-chemischen Feldparameter der Schicht-, vor allem aber der Kluftquellen mit relativ hohen elektrischen Leitfähigkeiten und vergleichsweise niedrigeren Temperaturen deuten auf eine tieferreichende Zirkulation der Wässer hin.

Im oberen Abschnitt der Bergsturzablagerung findet $\mathrm{Zu}$ fluss in den Lenzangerbach aus dem Zwischenabfluss statt. Eine gering durchlässige Schicht führt zur Ausbildung zahlreicher kleiner stehender Gewässer, die ausschließlich von Niederschlägen gespeist werden und nur langsam in den Bergsturz infiltrieren. In tieferen Abschnitten kommt es dagegen zu einer Dotation des Lenzangerbaches in den Grundwasserkörper. Eine von Bichler et al. (2016) kartierte Moräne, die dem Bergsturzmaterial bereichsweise auflagert, tritt erst in topographisch tieferen Lagen auf und spielt daher keine Rolle für die hydraulischen Eigenschaften des Bergsturzkörpers im oberen gering durchlässigen Bereich. Vielmehr sind Verwitterungsprozesse in den von Glimmerschiefern dominierten Bereichen für die Bildung einer geringmächtigen abdichtenden Schicht verantwortlich. In den tieferen Bereichen, die von Blockwerk aus Kalkglimmerschiefern dominiert werden, wird eine abdichtende Verwitterungsschwarte offenbar nicht flächig ausgebildet. Das zeigt sich auch an den im unteren Abschnitt weit weniger häufig auftretenden Stillgewässern.

\section{Schlussfolgerung}

Ziel der hier vorgestellten Studie war es, ein typisches inneralpines Gebiet in einem kleinräumigen Maßstab detailliert geologisch-hydrogeologisch zu untersuchen und daraus einige allgemein gültige qualitative Aussagen zur Hydrogeologie rund um das Tauernfenster ableiten zu können. Aus den Geländeaufnahmen lassen sich einige generelle Aussagen zu den hydrogeologischen Eigenschaften alpiner Einheiten, zu den auftretenden Quelltypen und zu deren Charakteristik ableiten. Vor allem die elektrische Leitfähigkeit erweist sich trotz der gering löslichen Lithologien im Untersuchungsgebiet als geeigneter Parameter, um unterschiedliche Fließsysteme zu identifizieren. So zeigen die als Schicht- oder Kluftquellen identifizierten Quellaustritte deutlich höhere elektrische Leitfähigkeiten als jene Wässer, die Schuttquellen zugeordnet werden. Die elektrischen Leitfähigkeiten der Stauquellen variieren dagegen sehr stark.
Die im Rahmen dieser Studie durchgeführten Stichtagsmessungen sind jedoch nur orientierender Natur. Konkrete Aussagen zur Dynamik der beteiligten Fließsysteme, z. B. auch zur einleitend angesprochenen Frage der Zirkulationstiefen innerhalb der nicht verkarstungsfähigen Festgesteinsaquifere setzen die Sammlung von Zeitreihendaten über Quantität und Qualität der Quellwässer voraus. Schüttungsganglinien, Zeitreihen hydrochemischer Parameter und stabiler Isotope über möglichst mehrere Jahre sind erforderlich, um das System im Detail zu erfassen und die hier vorgestellte erste Klassifizierung zu verifizieren.

Danksagung Wir bedanken uns bei Sebastian Pfleiderer und einem weiteren anonymen Gutachter sowie bei Gerfried Winkler als Editor für die wertvollen Anregungen zur Verbesserung des Manuskripts.

Funding Open access funding provided by Paris Lodron University of Salzburg.

Open Access Dieser Artikel wird unter der Creative Commons Namensnennung 4.0 International Lizenz veröffentlicht, welche die Nutzung, Vervielfältigung, Bearbeitung, Verbreitung und Wiedergabe in jeglichem Medium und Format erlaubt, sofern Sie den/die ursprünglichen Autor(en) und die Quelle ordnungsgemäß nennen, einen Link zur Creative Commons Lizenz beifügen und angeben, ob Änderungen vorgenommen wurden.

Die in diesem Artikel enthaltenen Bilder und sonstiges Drittmaterial unterliegen ebenfalls der genannten Creative Commons Lizenz, sofern sich aus der Abbildungslegende nichts anderes ergibt. Sofern das betreffende Material nicht unter der genannten Creative Commons Lizenz steht und die betreffende Handlung nicht nach gesetzlichen Vorschriften erlaubt ist, ist für die oben aufgeführten Weiterverwendungen des Materials die Einwilligung des jeweiligen Rechteinhabers einzuholen.

Weitere Details zur Lizenz entnehmen Sie bitte der Lizenzinformation auf http://creativecommons.org/licenses/by/4.0/deed.de.

\section{Literatur}

Alber, J.: Bericht 1997 über geologische Aufnahmen im Penninikum des Hüttwinkltales auf Blatt Rauris. Jhb. Geol. Bundesanst. 141, 297-302 (1998)

Banks, E.W., Simmons, C.T., Love, A.J., Cranswick, R., Werner, A.D., Bestland, E.A., Wood, M., Wilson, T.: Fractured bedrock and saprolite hydrogeologic controls on groundwater/surface-water interaction: a conceptual model (Australia). Hydrogeol. J. 17, 1969-1989 (2009)

Bense, V.F., Gleeson, T., Loveless, S.E., Bour, O., Scibek, J.: Fault zone hydrogeology. Earth Sci. Rev. 127, 171-192 (2013)

Bichler, M., Reindl, M., Reitner, J., Drescher-Schneider, R., Wirsig, C., Christl, M., Hajdas, I., Ivy-Ochs, S.: Landslide deposits as stratigraphical markers for a sequence-based glacial stratigraphy: a case study of Younger Dryas system in the eastern Alps. Boreas 45, 537-551 (2016)

Bichler, M., Reindl, M.: Landscape evolution north of the Sonnblick during the Alpine Lateglacial. 159 S., unveröff. Masterarbeit, Universität Wien (2013)

Caine, J.S., Evans, J.P., Forster, C.B.: Fault zone architecture and permeability structure. Geology (Boulder) 24, 1025-1028 (1996)

Exner, C.: Erläuterungen zur Geologischen Karte der Umgebung von Gastein. Geologische Bundesanstalt Wien, Wien (1957) 
Fellner, D.: Massenbewegungen im Bereich Bucheben im Hüttwinkltal bei Rauris (Salzburg, Österreich). Jhb. Geol. Bundesanst. 136(2), 307-313 (1993)

Fiori, A., Russo, D., Di Lazzaro, M.: Stochastic analysis of transport in hillslopes: Travel time distribution and source zone dispersion. Water Resour. Res. 45, W8435 (2009)

Günther, W., Paar, W. (Hrsg.): Schatzkammer Hohe Tauern - 2000 Goldbergbau. Anton Pustet, Salzburg, München (2000)

Hilberg, S., Kreuzer, M.: Identification of a deep flow system in a dolomitic alpine aquifer-case study Wimmerbauern spring, Bad Ischl. Austrian J. Earth. Sci. 106(1), 16-25 (2013)

Hilberg, S., Riepler, F.: Interaction of various flow systems in small alpine catchments: conceptual model of the upper Gurk valley aquifer, Carinthia, Austria. Hydrogeol J 24(5), 1231-1244 (2016)

Hilberg, S., Brandstätter, J., Glück, D.: $\mathrm{CO}_{2}$-partial pressure and calcite saturation in springs - useful data for identifying infiltration areas in mountainous environments. Environ. Sci. Process. Impacts 15, 823-832 (2013)

Hobiger, G., Kollmann, W.H.: Hydrogeologie. In: Schuster, R., Pestal, G., Reitner, J. (Hrsg.) Erläuterungen zu Blatt 182 Spittal an der Drau. Geologische Bundesanstalt Wien, Wien (2006)

Hottinger, A.: Geologie der Gebirge zwischen der Sonnblick-HocharnGruppe und dem Salzachtal in den östlichen Hohen Tauern. 123 S., unveröff. Ph.D. Thesis. Eidgenössische Technische Hochschule Zürich (1935)

Jones, D.B., Harrison, S., Anderson, K., Whalley, W.B.: Rock glaciers and mountain hydrology: a review. Earth Sci. Rev. 193, 66-90 (2019)

Land Salzburg: SAGISonline (2014). https://www.salzburg.gv. at/sagisonline/(S(mbmxpdye0t2pud3eith5qbyq))/init.aspx? karte $=$ default $\&$ geojuhuschema $=$ Adressen $/$ Namensgut $\&$ defaultlogo=sagis, Zugegriffen: 30. Sept. 2014

Lauber, U., Kotyla, P., Morche, D., Goldscheider, N.: Hydrogeology of an Alpine rockfall aquifer system and its role in flood attenuation and maintaining baseflow. Hydrol. Earth Syst. Sci. 18, 4437-4452 (2014)

Masset, O., Loew, S.: Hydraulic conductivity distribution in crystalline rocks, derived from inflows to tunnels and galleries in the central Alps, Switzerland. Hydrogeol J 18, 863-891 (2010)

Matura, A.: Zur Geologie des Türchlwand-Kramkogel-Gebietes: (SE Wörth im Rauristal, Salzburg). Mitt. Ges. Geol. Bergbaustud. Wien 17, 87-126 (1966)

Paar, W., Gruber, F., Günther, W. (Hrsg.): Das Buch vom Tauerngold. Anton Pustet, Salzburg, München (2006)

Pauritsch, M., Wagner, T., Winkler, G., Birk, S.: Investigation of groundwater flow components in an Alpine relict rock glacier (Austria) using a numerical model. Hydrogeol J 25, 371-383 (2017)
Pestal, G., Hejl, E., Braunstingl, R. Schuster, R.: Erläuterungen zur Geologischen Karte von Salzburg 1:200 000. 162 S. Geologische Bundesanstalt Wien (2009)

Reitner, J.M., Lang, M., van Husen, D.: Deformation of high slopes in different rocks after Würmian deglaciation in the Gailtal (Austria). Quat. Int. 18, 43-51 (1993)

Ronchetti, F., Borgatti, L., Cervi, F., Gorgoni, C., Piccinini, L., Vincenzi, V., Corsini, A.: Groundwater processes in a complex landslide, northern Apennines, Italy. Nat. Hazards Earth Syst. Sci. 9, 895-904 (2009)

Salzburg AG: Wo unsere saubere Energie herkommt - Kraftwerksgruppe Gasteiner Tal. https://www.salzburg-ag.at/content/dam/ web18/dokumente/unternehmen/Kraftwerke-GasteinerTal.pdf (2018). Zugegriffen: 10. Sept. 2019

Schmid, S.M., Scharf, A., Handy, M.R., Rosenberg, C.L.: The Tauern Window (Eastern Alps, Austria): a new tectonic map, with crosssections and a tectonometamorphic synthesis. Swiss Journal of Geosciences (2013)

Strauhal, T., Loew, S., Holzmann, M., Zangerl, C.: Detailed hydrogeological analysis of a deep-seated rockslide at the Gepatsch reservoir (Klasgarten, Austria). Hydrogeol. J. 26, 349-371 (2015)

Vallet, A., Bertrand, C., Mudry, J., Bogaard, T., Fabbri, O., Baudement, C., Regent, B.: Contribution of time-related environmental tracing combined with tracer tests for characterization of a groundwater conceptual model: a case study at the Sechilienne landslide, western Alps (France). Hydrogeol. J. 23, 1761-1779 (2015)

von Poschinger, A.: Instabile Talflanken in Kristallingesteinen und ihre geologischen Ursachen, dargestellt am Beispiel des oberen Hüttwinkltales (Land Salzburg, Östereich). 170 S., unveröff. PhD thesis, Technische Universität München (1986)

Welch, L.A., Allen, D.M.: Hydraulic conductivity characteristics in mountains and implications for conceptualizing bedrock groundwater flow. Hydrogeol. J. 22, 1003-1026 (2014)

Winkler, G., Kurz, W., Hergarten, S., Kiechl, E.: Hydraulische Charakterisierung von Störungskernzonen in kristallinen Festgesteinen am Beispiel der Talhof-Störung (Ostalpen). Grundwasser 15(1), 59-68 (2010)

Winkler, G., Wagner, T., Pauritsch, M., Birk, S., Kellerer-Pirklbauer, A., Benischke, R., Leis, A., Morawetz, R., Schreilechner, M., Hergarten, S.: Identification and assessment of groundwater flow and storage components of the relict Schöneben Rock Glacier, Niedere Tauern Range, Eastern Alps (Austria). Hydrogeol. J. 24, 937-953 (2016)

Hinweis des Verlags Der Verlag bleibt in Hinblick auf geografische Zuordnungen und Gebietsbezeichnungen in veröffentlichten Karten und Institutsadressen neutral. 